\title{
Nutritional Metabonomics: An Approach to Promote Personalized Health and Wellness
}

\author{
Sebastiano Collino, François-Pierre J. Martin, Sunil Kochhar, and Serge Rezzi
}

\begin{abstract}
Nutritional research has emerged in the last century from the study of nutrients as a means of nourishment to the general population to the quest for wellness improvement through specific food components. Advances in nutrigenomics technologies have allowed nutrition scientists to be for the first time at the forefront of nutritional research. Such advances have given them the ability to discern new vital scientific discoveries specifically for the development of new tailored dietary patterns. In this, nutritional metabonomics has rapidly evolved into a very powerful bioanalytical tool able to assess multi-parametric metabolic responses of living organisms to specific dietary interventions. Nutritional metabonomics therefore provides a systematic approach through the comprehensive analysis of metabolites aiming today at the quest for homeostatic balance which is dependent not only on the host but also on the crucial metabolic interactions with microbial symbionts.
\end{abstract}

Keywords: Gut microbiota · Host metabolism · Metabotypes · Nutritional metabonomics ·

Personalized nutrition

\section{Introduction}

Over the past years, changes in diet and lifestyle have rapidly evolved with a direct influence on the health and nutritional status of populations. Dietary adjustments and their alteration not only influence present health but it is now known that they determine whether or not individuals will develop chronic diseases such as cancer, diabetes, and/or cardiovascular diseases later in life. It has been estimated that by 2020 chronic disease in developing countries will account for almost three-quarters of mortality worldwide with $75 \%$ of deaths due to stroke, and $70 \%$ of deaths due to diabetes. In addition, an important challenge that nutrition research faces today is the complexity and variability of different food matrices that may contain a high number of bioactive compounds, carbohydrates, fats and proteins to essential micro-

\footnotetext{
${ }^{\star}$ Correspondence: Dr. S. Rezzi Nestlé Research Center

Metabonomics \& Biomarkers

P.O. Box 44, CH-1000 Lausanne 26

Tel.: +41217859165

Fax: +41217859486

E-mail: Serge.Rezzi@rdls.nestle.com
}

nutrients such as vitamins and minerals. Nutritional research has also shifted from the typical measurements of a few, but key, physiological descriptors, to large-scale screening of molecular processes at different levels of biological organisation, from gene to mRNA, to proteins and enzymes, and to metabolic pathways. [1] Studies on the individual and population scale are often a very complicated task because biological processes are under the influence of numerous intrinsic and extrinsic parameters such as environmental stressors, drugs, diet, lifestyle, stress, and microbiome modulation. ${ }^{[2,3]}$

Metabonomics, in particular, measures and monitors metabolite concentrations in cells, tissues, and complex biological systems ${ }^{[2,4,5]}$ providing the ability to capture subtle changes in biological processes as a result of different nutritional effects. ${ }^{[6-8]}$ Because specific physiological states, gene expression and environmental stressors can cause changes in the steady state of a biological system, monitoring the resulting metabolic variations provides unique insights into physiological regulatory processes. By the comprehensive study of low molecular weight compounds $(<1500 \mathrm{Da})$ in biofluids (plasma/serum and urine) and tissues, ${ }^{[9]}$ metabonomics assure the characterization of individual metabolic phenotypes, or metabotypes. Metabonomics employs mainly two analytical techniques based on ${ }^{1} \mathrm{H}$ nuclear magnetic resonance (NMR) spectroscopy, and mass spectrometry coupled to gas/high performance liquid chromatography (GC/MS and LC/ MS) (Fig. 1). Both methods are then used to generate metabolic profiles, from which meaningful biological information is recovered using advanced statistical tools.

Recent applications of metabonomics in nutritional research are reviewed here with the specific aim to ameliorate our current knowledge in foods and nutrient functionalities to reveal the biological activities of different food components. ${ }^{[10]}$

\section{Nutrimetabonomics: A Tool to Decipher Specific Food Intake Imprinting while Monitoring Different Physiological States}

Besides initial and successful applications to screen and diagnose for certain diseases ${ }^{[11]}$ in the last 5-10 years, metabonomics has been successfully applied to nutritional studies. ${ }^{[12]}$ Here the term 'nutrimetabonomics' clearly embodies the novel goal to study the metabolic signatures of specific food habits elucidating the molecular mechanisms behind individual metabolic responses. Indeed, one of the most important challenges of nutrition research today is to determine the biochemical effects of diets and foods on individuals and to discern the underlying mechanism of action of biologically active food constituents.

One of the first studies that applied metabonomic approaches in human nutritional experiments was directed to monitor the effects of supplementing the diet with soy extracts. ${ }^{[13]}$ Clear differences in pre-menopausal women in the plasma lipoprotein, amino acid, and carbohydrate profiles were observed following soy intervention, indicating an alteration in energy 


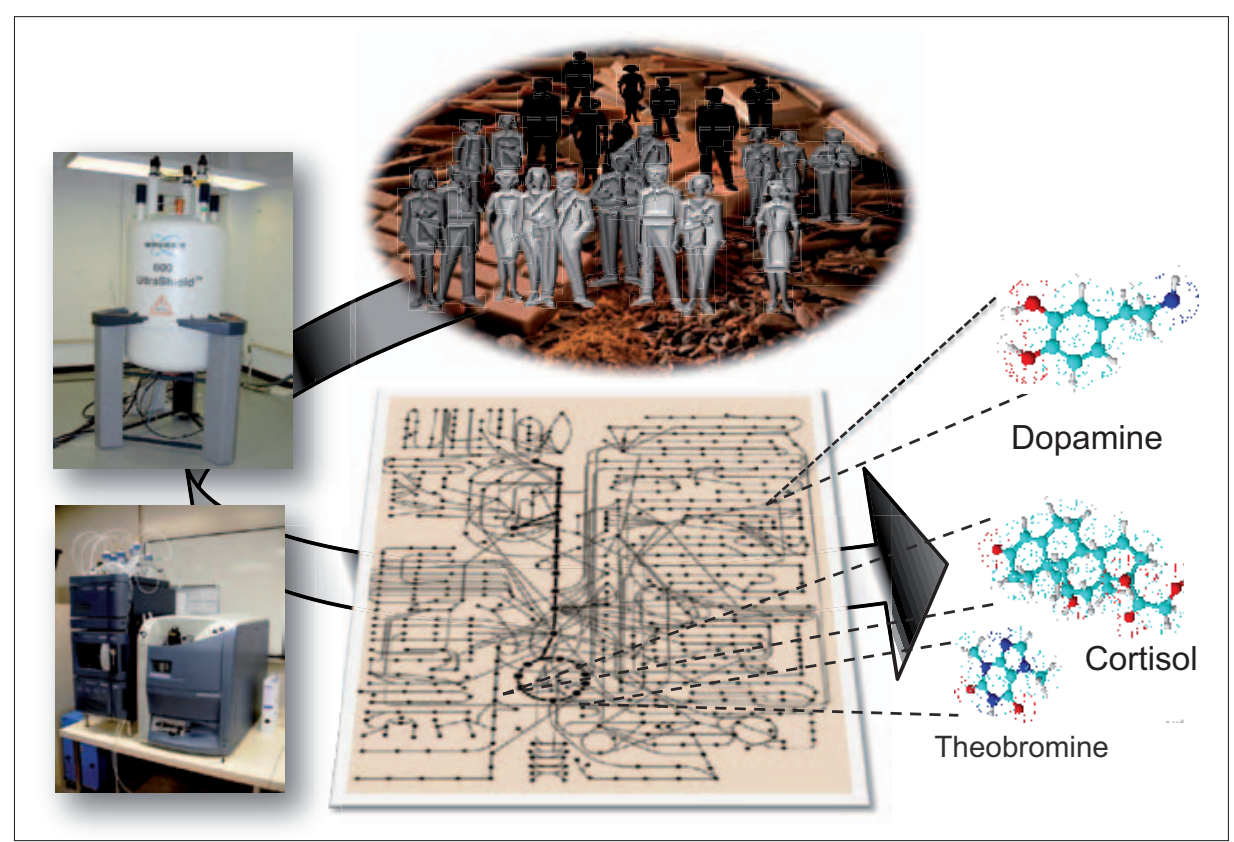

Fig. 1. Scheme describing a typical NMR (Bruker $600 \mathrm{MHz}$ ) and MS (Waters Acquity Ultra Performance Liquid Chromatograph) based metabonomic analytical platform. NMR offers a holistic profiling of a wide range of metabolites with no a priori selection, while MS methods are commonly employed for global and targeted profiling. Both techniques are jointly employed. Multivariate statistical techniques are then used for encoding the complex metabolic profiles, identification of biomarkers to be ultimately used for the elaboration of biological outcome. Chemical structures of selected compounds were drawn with ACDLab software (www.acdlabs.com).

metabolism. A metabonomic strategy was also applied to study the human response to chamomile tea ingestion. ${ }^{[14]}$ The author demonstrated that depletion of creatinine and elevated excretion of hippurate, and glycine were associated with chamomile intake. Law et al. investigated the metabolic changes after intake of green tea in human urine, showing that a number of metabolites involved in the citric acid cycle, glucose and amino acid metabolism were affected immediately after tea intake.[15] A strategy to assess the metabolic phenotypes following nutritional intervention, based on polyphenols/black tea consumption, in a full cross-over population study was also revealed by applying an integrated metabolomic and nutrikinetic approach. ${ }^{[16]}$ Here, variation in urinary extraction of several gut microbial metabolites, including hippuric acid, 4-hydroxyhippuric acid, and 1,3-dihydrophenyl-2-O-sulfate, were revealed after black tea consumption. In addition, nutrikinetic properties and response of selected phenolic acids were also investigated by the authors with interesting behaviour in urinary excretion revealed among and within subjects.

More recently, an exploratory study in healthy subjects compared the shortterm impact of a diet rich in commercially available whole grain cereal products with a diet rich in refined grain cereal products revealing possible effects on plasma markers of cardiovascular and gastrointestinal health. ${ }^{[17]}$ Additionally, nutrimetabonomic studies have shown that specific dietary metabolic phenotypes, or metabotypes, in both human host basal metabolism and gut microbiota activity, are closely related to specific individual dietary preferences. ${ }^{[18]}$ Metabolic profiling of urine revealed that 'chocolate lovers' have a specific energy metabolism and harboured a gut microbiota with different activities. Additionally, a combined NMR and MS approach was employed to study the metabolic response due to dark chocolate consumption, with key emphasis on stress metabolism ${ }^{[19]}$ (Fig. 1). In this study, the authors monitored the effect of consuming regularly $40 \mathrm{~g}$ dark chocolate in healthy volunteers classified according to their level of anxiety using validated psychological questionnaires. Human subjects with higher anxiety trait showed a distinct metabolic profile indicative of a different energy homeostasis (lactate, citrate, succinate, trans-aconitate, urea, proline), hormonal (adrenaline, DOPA, 3-methoxy-tyrosine) and gut microbial metabolism (methylamines, $p$-cresol sulfate, hippurate). Daily dark chocolate consumption reduced the urinary excretion of the stress hormone cortisol and catecholamines while partially normalizing stress-related differences in energy metabolism (glycine, citrate, trans-aconitate, proline, $\beta$-alanine) and gut microbial activities towards the levels observed in subjects with low anxiety traits. The study provides evidence that a daily consumption of dark chocolate over a period of two weeks is sufficient to modify the metabolism of free living and healthy human subjects.

The capability to estimate specific dietary intake compounds in individuals is also an important asset of nutritional studies. Indeed, an approach to validate biomarkers of intake among polyphenol-rich food consumption, in the free living population, and urinary excretion of phenolic compounds was directed by mass spectrometry. ${ }^{20]}$ Remarkably, the authors found that the consumption of fruit and fruit juices were positively correlated to gallic acid, 4-Omethylgallic acid, isorhamnetin, kaempferol, hesperetin, naringenin and phloretin, whereas, for example, coffee consumption was positively correlated to caffeic and chlorogenic acids excretion. Most recently, to reveal the effects of diet on health, proline betaine was discerned as a putative biomarker of citrus consumption in free living individuals. ${ }^{[21]}$

Nutritional studies are also today closely applied to decipher the mechanisms behind different physiological states. For example, the complex molecular mechanisms behind obesity-associated insulin resistance were discerned by nutritional metabonomic applications. ${ }^{[22]}$ By applying a multi-analytical metabonomics approach the authors were able to reveal specific endocrine, inflammatory, and physiological differences between obese and lean humans. Here, it was shown that the context of a dietary pattern that includes high fat consumption, branch chain amino acids (BCAA) contribute to the development of obesity-associated insulin resistance. Levels of the BCAAs valine and leucine/isoleucine were $20 \%$ and $14 \%$ higher, respectively, in obese compared to lean subjects. To confirm this hypothesis, rats were fed on high-fat (HF), HF with supplemented BCAA (HF/BCAA), or standard chow (SC) diets. Interestingly, despite having reduced food intake and a low rate of weight gain equivalent to the $\mathrm{SC}$ group, $\mathrm{HF} /$ BCAA rats were as insulin resistant as $\mathrm{HF}$ rats. Most recently, a UPLC-Q-TOF MSbased metabolomics approach revealed a cluster of obesity-associated changes in metabolites, which included three lysoPC species (C14:0, C18:0, and C18:1), specific amino acids (two BCAA, phenylalanine and tryptophan), L-carnitine, and acylcarnitines (propionyl-, butyryl-, and hexanoyl-carnitine). ${ }^{[23]}$ Waldram et al. applied metabonomic approaches in combination to microbial profiling to investigate the transgenomic metabolic interactions and their relationship to obesity. ${ }^{[24]}$ Urine and plasma samples from lean and obese strains of Zucher rats were analyzed by ${ }^{1} \mathrm{H}-\mathrm{NMR}$ spectroscopy, whilst faecal microbial composition was investigated by fluorescence in situ hybridization and 
denaturing gel electrophoresis. Lean and obese animals displayed specific metabolic phenotypes linked to their individual microbiomes. Specifically, higher levels of urinary isoleucine, leucine, acetate and higher plasma LDL and VLDL levels were seen in the obese strain.

\section{Nutrimetabonomics: Measuring the Host-Gut Microbiota Metabolic Cross Talk}

The homeostatic balance of our human organisms also depends on the complex metabolic interactions with the gut microbial ecosystem. ${ }^{[25]}$ Since recent evidence described how gut microbial species and metabolic capabilities may impact upon health and predisposition to various diseases such as inflammatory bowel diseases and colon cancer, ${ }^{[25-27]}$ there is clearly an important role of understanding these interactions for the development of tailored nutritional solutions.

Increasing awareness on the nature of the gut microbiome-host interactions tends to highlight that the host controls the microbiome community structure, a process that has evolved to attain specific benefits ranging from protection, nutrition to physiology. ${ }^{28]}$ The measurement of the gut microbial populations and metabolism is generally confined to faecal samples, which represent only a limited range of microbial species and activities within the colon, in part due to the elevated colonic absorption of bacterial metabolites. ${ }^{[29]}$ However, such analyses provided some essential information - as yet incomplete - with regards to the contributions of the gut microbiota to energy recovery, lipid and bile acid metabolism. ${ }^{[30]}$ Saric et al. analyzed faecal extracts to compare metabolites profiles from human, mice, and rats.[31] While some faecal compounds were common to the three species, ${ }^{1} \mathrm{H}$ NMR analysis generated a unique metabolic profile specific to each species. Humans displayed greater inter-individual variations, with glycerol and malonate being specific compounds in respect to the other two species. Moreover, human faecal water extracts displayed a deep inter-individual variation, suggesting greater difference in genetic and environmental diversity.

The symbiotic relationships among animals and their gut has been investigated by Martinetal. whodescribed atop-down view model of the effects of different gut microbiome on murine metabolic profiles. ${ }^{[32]}$ The authors reported that inoculation of germ-free mice with a simplified model of human baby microbiota modifies the physiology of the murine host towards pre-pathological state. It was also demonstrated that gut microbiotal activities have specific impacts on specific intestinal epithelial biochemistry, influencing the global metabolic host phenotypes.[33] The metabolic relationship between gut microbiota and their effects on the biochemical profiles of intestinal tissues was also recently investigated. ${ }^{[34]}$ Indeed, the metabolic impact of the gut microbiota on the biochemical composition of intact intestinal tissue (duodenum, jejunum, ileum, proximal and distal colon) from four different microbiome mouse models (conventional, conventionalized, human baby microbiota (HBM) colonized with probiotic supplementation, and HBM colonized with symbiotic) were analyzed by solid-state NMR spectroscopy. Interestingly, supplementation of HBM mice with probiotics and synbiotics resulted in different metabolic signatures in the proximal and distal colon, displaying different concentrations of bacterial products.

Modern nutritional research is also aiming at the characterization of health maintenance through the modulation of the gut microbial functional ecology by the use of prebiotics and probiotics. Prebiotics are very important in infant nutrition, as formula-fed infants have lower numbers of bifidobacteria compared to breast-fed infants. ${ }^{[35]}$ Probiotic and prebiotic products were also tested on elderly people where improvements in intestinal microbial composition and immune functions were evident. ${ }^{[36-38]}$

By adapting the gut functional ecology with pro-, pre-, and synbiotics, the depth of gut microbiome modulations on the host biochemistry was explored by Martin et al. ${ }^{[39]}$ Here metabonomics was able to captured metabolic changes in selected biological compartments, biofluids and liver, which were correlated with modulation in microbial population. These microbial effects were associated with changes in various host metabolic pathways including gluconeogenesis, amino acids, methylamine, and lipid metabolism. Single preand probiotics and their combinatorial effects on the metabolic status of germ-free mice were also analyzed by metabonomics means during the establishment of a simplified model of human microbiota. Specifically, it was found that galactosyl-oligosaccharide prebiotics strongly reduced lipogenesis, triacylglycerol incorporation into lipoproteins, and triglyceride concentration in the liver and the kidney. Prebiotic modulation of the gut microbiota also altered transmethylation metabolic pathways in the liver and in the pancreas, with inferred effects on the control of glucose metabolism and insulin sensitivity.

Recently, metabonomics was employed in a series of animal and human studies to provide compelling evidence of a functional link between systemic levels of circulating metabolites with gut microbiome activities and populations. The evaluation of the relative contribution of the gut microbiota to the host metabolism was assessed by investigating the effects of the antibiotic vancomycin, which impact specifically microbial metabolism since it does not have systemic absorption. ${ }^{[40]}$ Here, reduced urinary excretion of gut microbial co-metabolites induced by the disruption of the microbial functional ecology described the interdependent microbial-mammalian co-metabolism of waste products derived from gut microbial metabolism and their elimination by the host via the urine. Wikoff et al. have shown the full influence of the microbiome on mammalian plasma biochemistry. ${ }^{[41]}$ Here, a MS metabolic approach revealed significant differences among circulating molecules between germ-free mice and conventional mice, with some of these metabolites appearing exclusively in the presence of gut microbiota. For example, production of indole-2-propionic acid was shown to be completely dependent on the presence of gut microflora and could be established by colonization with the bacterium Clostridium sporogenes. More recently, a metabonomics study in mice revealed a significant association between changes of the gut microbiome and specific faecal metabotypes collected from four groups of animals (conventional and conventionalized mice, gnotobiotic animals and supplemented with Lactobacillus paracase $i$ with and without prebiotics). ${ }^{[42]}$ In addition, an interesting model to map the microbial-host metabolic interactions was applied by Li et al. ${ }^{[43]}$ where a combined spectroscopic, microbiomic, and multivariate statistical approach was used to analyze faeces and urine samples from seven Chinese individuals with correlations among specific host metabolic pathways and variation of gut microbiota. For example, $F$. prausnitzii was statistically linked with the rate of urinary excretion of dimethylamine, taurine, lactate, glycine, 2-hydroxyisobutyrate, glycolate, 3,5-hydroxylbenzoate, and 3 -aminoisobutyrate.

It is clear that nowadays strategies to discern the potential impacts of pro- and prebiotic products on the composition of the gut microbiota and the systematic effects on the host immune systems and metabolism are well initiated. However proper human clinical studies are still needed to determine the specific effects of each probiotic strain and prebiotic. Undeniably, the elucidation of these mechanisms will bring at the design of novel and enhanced functional foods with new host health amelioration targeted approaches.

\section{Conclusion}

One of the greatest challenges in modern metabonomics research is to decipher how changes in the environment and lifestyle conditions regulate human physiol- 
ogy. The characterization of the metabotype of individuals could open access to important information on dietary variations in man and on the degree of response to dietary modulations. It is therefore expected that nutrition and health will commonly aim in the immediate future at the optimization of food products specifically tailored to different individual needs. Metabonomics will provide the required instruments to monitor the metabolic health of consumers, maintaining homeostatic balance where adjusting the diet according to the health status will be one of the projected benefits.

Received: April 6, 2011

[1] L. Tiret, Pro. Nutr. Soc. 2002, 61, 457.

[2] J. K. Nicholson, I. D. Wilson, Nat. Rev. Drug Discov. 2003, 2, 668.

[3] J. K. Nicholson, J. C. Lindon, E. Holmes, Xenobiotica. 1999, 29, 1181.

[4] J. K. Nicholson, J. C. Lindon, Nature 2008, 455 , 1054.

[5] E. Holmes, I. D. Wilson, J. K. Nicholson, Cell 2008, 134, 714.

[6] J. J. Keurentjes, Curr. Opin. Plant Biol. 2009, $12,223$.

[7] K. Saito, F. Matsuda, Annu. Rev. Plant Biol. 2010, 61, 463 .

[8] W. Weckwerth. Annu. Rev. Plant Biol. 2003, 54, 669.

[9] O. Beckonert, M. Coen, H. C. Keun, Y. Wang, T. M. Ebbels, E. Holmes, J. C. Lindon, J. K. Nicholson, Nat. Protoc. 2010, 5, 1019.

[10] M. Kussmann, S. Rezzi, H. Daniel, Curr. Opin. Biotechnol. 2008, 19, 83.

[11] V. P. Makinen, P. Soininen, C. Forsblom, M Parkkonen, P. Ingman, K. Kaski, P. H. Groop, M. la-Korpela, Mol. Syst. Biol. 2008, 4, 167.

[12] S. Rezzi, F. P. Martin, S. Kochhar, Ernst. Schering. Found. Symp. Proc. 2007, 251.

[13] K. S. Solanky, N. J. Bailey, B. M. BeckwithHall, A. Davis, S. Bingham, E. Holmes, J. K. Nicholson, A. Cassidy, Anal. Biochem. 2003, 323, 197.

[14] Y. Wang, H. Tang, J. K. Nicholson, P. J. Hylands, J. Sampson, E. Holmes. J. Agr. Food Chem. 2004, 53, 191.
[15] W. S. Law, P. Y. Huang, E. S. Ong, C. N. Ong, S. F. Li, K. K. Pasikanti, E. C. Chan. Rapid Commun. Mass Spectrom. 2008, 22, 2436.

[16] E. J. van Velzen, J. A. Westerhuis, J. P. van Duynhoven, F. A. van Dorsten, C. H. Grun, D. M. Jacobs, G. S. Duchateau, D. J. Vis, A. K. Smilde. J. Proteome Res. 2009, 8, 3317.

[17] A. B. Ross, S. J. Bruce, A. Blondel-Lubrano, S. Oguey-Araymon, M. Beaumont, A. Bourgeois, C. Nielsen-Moennoz, M. Vigo, L. B. Fay, S. Kochhar, R. Bibiloni, A. C. Pittet, S. EmadyAzar, D. Grathwohl, S. Rezzi, Br. J. Nutr. 2011, 105, 1492.

[18] S. Rezzi, Z. Ramadan, F. P. Martin, L. B. Fay, B. P. van, J. C. Lindon, J. K. Nicholson, S. Kochhar, J. Proteome Res. 2007, 6, 4469.

[19] F. P. Martin, S. Rezzi, E. Pere-Trepat, B. Kamlage, S. Collino, E. Leibold, J. Kastler, D. Rein, L. B. Fay, S. Kochhar, J. Proteome Res. 2009, 8, 5568 .

[20] L. I. Mennen, D. Sapinho, H. Ito, S. Bertrais, P. Galan, S. Hercberg, A. Scalbert, Br. J. Nutr. 2006, 96, 191.

[21] S. S. Heinzmann, I. J. Brown, Q. Chan, M. Bictash, M. E. Dumas, S. Kochhar, J. Stamler, E. Holmes, P. Elliott, J. K. Nicholson, Am. J. Clin. Nutr. 2010, 92, 436.

[22] C. B. Newgard, J. An, J. R. Bain, M. J. Muehlbauer, R. D. Stevens, L. F. Lien, A. M. Haqq, S. H. Shah, M. Arlotto, C. A. Slentz, J. Rochon, D. Gallup, O. Ilkayeva, B. R. Wenner, W. S. Yancy, Jr., H. Eisenson, G. Musante, R. S. Surwit, D. S. Millington, M. D. Butler, L. P. Svetkey, Cell Metab. 2009, 9, 311

[23] J. Y. Kim, J. Y. Park, O. Y. Kim, B. M. Ham, H. J. Kim, D. Y. Kwon, Y. Jang, J. H. Lee, J. Proteome Res. 2010, 9, 4368.

[24] A. Waldram, E. Holmes, Y. Wang, M. Rantalainen, I. D. Wilson, K. M. Tuohy, A. L. McCartney, G. R. Gibson, J. K. Nicholson, J. Proteome Res. 2009, 8, 2361.

[25] L. Dethlefsen, M. Fall-Ngai, D. A. Relman, Nature 2007, 449, 811.

[26] J. K. Nicholson, E. Holmes, J. C. Lindon, I. D. Wilson, Nat. Biotechnol. 2004, 22, 1268.

[27] C. Dunne, Inflamm. Bowel. Dis. 2001, 7, 136.

[28] J. K. Nicholson, E. Holmes, I. D. Wilson, Nat. Rev. Microbiol. 2005, 3, 431.

[29] J. H. Cummings, G. T. Macfarlane, J. Appl. Bacteriol. 1991, 70, 443.

[30] F. P. Martin, M. E. Dumas, Y. Wang, C. Legido-Quigley, I. K. Yap, H. Tang, S. Zirah, G. M. Murphy, O. Cloarec, J. C. Lindon, N. Sprenger, L. B. Fay, S. Kochhar, B. P. van,
E. Holmes, J. K. Nicholson, Mol. Syst. Biol. 2007, 3, 112.

[31] J. Saric, Y. Wang, J. Li, M. Coen, J. Utzinger, J. R. Marchesi, J. Keiser, K. Veselkov, J. C. Lindon, J. K. Nicholson, E. Holmes, $J$. Proteome Res. 2008, 7, 352.

[32] F. P. Martin, Y. Wang, N. Sprenger, I. K. Yap, S Rezzi, Z. Ramadan, E. Pere-Trepat, F. Rochat, C. Cherbut, B. P. van, L. B. Fay, S. Kochhar, J. C. Lindon, E. Holmes, J. K. Nicholson, Mol. Syst. Biol. 2008, 4, 205.

[33] S. P. Claus, T. M. Tsang, Y. Wang, O. Cloarec, E. Skordi, F. P. Martin, S. Rezzi, A. Ross, S. Kochhar, E. Holmes, J. K. Nicholson, Mol. Syst. Biol. 2008, 4, 219.

[34] F. P. Martin, Y. Wang, I. K. Yap, N. Sprenger, J. C. Lindon, S. Rezzi, S. Kochhar, E. Holmes, J. K. Nicholson, J. Proteome Res. 2009, 8, 3464.

[35] G. Boehm, G. Moro, J. Nutr. 2008, 138, 1818S.

[36] J. Vulevic, A. Drakoularakou, P. Yaqoob, G. Tzortzis, G. R. Gibson. Am. J. Clin. Nutr. 2008 $88,1438$.

[37] E. J. Schiffrin, D. R. Thomas, V. B. Kumar, C. Brown, C. Hager, M. A. van't Hof, J. E. Morley, Y. Guigoz. J. Nutr. Health Aging 2007, 11, 475.

[38] E. Nova, J. Warnberg, S. Gomez-Martinez, L. E. Diaz, J. Romeo, A. Marcos. Br. J. Nutr. 2007, 98 Suppl 1, S90-S95.

[39] F. P. Martin, N. Sprenger, I. K. Yap, Y. Wang, R. Bibiloni, F. Rochat, S. Rezzi, C. Cherbut, S. Kochhar, J. C. Lindon, E. Holmes, J. K. Nicholson, J. Proteome Res. 2009, 8, 2090.

[40] I. K. Yap, J. V. Li, J. Saric, F. P. Martin, H Davies, Y. Wang, I. D. Wilson, J. K. Nicholson, J. Utzinger, J. R. Marchesi, E. Holmes, J. Proteom. Res. 2008, 7, 3718.

[41] W. R. Wikoff, A. T. Anfora, J. Liu, P. G. Schultz, S. A. Lesley, E. C. Peters, G. Siuzdak. Proc. Natl. Acad. Sci. USA 2009, 106, 3698.

[42] F. P. Martin, N. Sprenger, I. Montoliu, S. Rezzi, S. Kochhar, J. K. Nicholson, J. Proteome Res. 2010, 9, 5284.

[43] M. Li, B. Wang, M. Zhang, M. Rantalainen, S Wang, H. Zhou, Y. Zhang, J. Shen, X. Pang, M. Zhang, H. Wei, Y. Chen, H. Lu, J. Zuo, M. Su, Y. Qiu, W. Jia, C. Xiao, L. M. Smith, S. Yang, E. Holmes, H. Tang, G. Zhao, J. K. Nicholson, L. Li, L. Zhao. Proc Natl. Acad. Sci. USA 2008 105, 2117. 